\title{
ELECTROCHEMICAL SENSORS FOR SOIL NUTRIENT DETECTION: OPPORTUNITY AND CHALLENGE
}

\author{
Jianhan Lin, Maohua Wang ${ }^{*}$, Miao Zhang, Yane Zhang, Li Chen \\ Key Laboratory of Modern Precision Agriculture System Integration, Ministry of Education, \\ Beijing, China, 100083 \\ ${ }^{1}$ Corresponding author, Address: Box 115, China Agricultural University, 17 Tsinghua East \\ Road, Beijing, 100083, China, Tel/Fax:+86-10-82377326, Email: wangmh@cau.edu.cn
}

\begin{abstract}
Soil testing is the basis for nutrient recommendation and formulated fertilization. This study presented a brief overview of potentiometric electrochemical sensors (ISE and ISFET) for soil NPK detection. The opportunities and challenges for electrochemical sensors in soil testing were discussed.
\end{abstract}

Keywords: $\quad$ ISE, ISFET, Soil Nutrient Detection, Nitrogen, Phosphorus, Potassium

\section{INTRODUCTION}

Over-application of fertilizers in China's agricultural production system has caused low fertilizer usage efficiency ( $35 \%$ in average, NBS, 2006), low agricultural product quality and serious environmental pollution, etc. One measure taken was to test soil for formulated fertilization.

A key in soil testing for formulated fertilization is to determine the amount of soil nutrients, followed by recommendation of nutrient needs and site-specific fertilization. Of the nutrients for crop growth, Nitrogen (nitrate: $\mathrm{NO}_{3}{ }^{-}$and ammonium: $\mathrm{NH}_{4}{ }^{+}$), Phosphorus (phosphate: $\mathrm{PO}_{4}{ }^{3-}$, hydrophosphate: $\mathrm{HPO}_{4}{ }^{2-}$ and dihydrophosphate: $\mathrm{H}_{2} \mathrm{PO}_{4}{ }^{-}$) and Potassium (potash: $\mathrm{K}^{+}$) are the most important elements. Conventional soil NPK testing methods have been generally performed by three steps: soil sampling, sample pretreatment and

Lin, J., Wang, M., Zhang, M., Zhang, Y. and Chen, Li., 2008, in IFIP International Federation for Information Processing, Volume 259; Computer and Computing Technologies in Agriculture, Vol. 2; Daoliang Li; (Boston: Springer), pp. 1349-1353. 
chemical analysis. To date, soil sampling is manually carried out in a field to obtain representative soil samples at a proper depth $(\sim 20 \mathrm{~cm})$. A vehiclebased hydraulic soil sampler (Wintex 1000, H.M. Agritech Aps, Denmark) was reported to achieve half-automated sampling in fields. Soil sample pretreatment is performed for the purpose of soil extracts through sequential processes: drying, crushing, sieving, extracting and filtering. Most processes can be carried out in batches, but are required to operate and connect by hand. Chemical analysis is handled by trained operators on special instruments to obtain the concentrations of soil nutrients.

So far, soil nutrient detection commonly uses optical measurement. In general, visible/ultraviolet spectrometry is employed for detecting nitrogen and phosphorus and flame spectrometry or atomic absorption spectrometry for potassium. The optical methods are reliable, but time-consuming, complex and high cost per test ( 150 Yuan/Sample). This resulted in the limitation of the number of soil samples tested for characterizing the spatial variability of soil nutrients in a field or fields. Therefore, novel soil nutrient detection methods are urgently needed. This study intended to give a brief review of potential electrochemical sensors and a summary of their challenges and opportunities in soil nutrient detection.

\section{ELECTROCHEMICAL SENSORS FOR SOIL NUTRIENT DETECTION}

An electrochemical sensor consists of an ion selective membrane, which selectively responds to a target ion, and a transducer, which transforms the reactions into detectable electrical signals. Ion Selective Electrode (ISE) and Ion Selective Field Effect Transistor (ISFET) are two types of commonlyused potentiometric electrochemical sensors for soil nutrient detection.

\subsection{Ion Selective Electrode}

The response mechanism of ISE method can briefly described by the Nernst equation as a change of an ISE's potential, compared with a reference electrode, is linear to the change of the ionic activity (in logarithmic units) of the target ion.

ISEs were reported to detect soil nitrate (Dahnke, 1971; Hansen, 1977), ammonium (Banwart, 1972; Simeonov, 1976) and potassium (Mei, 1982; Wang, 1992). To date, no promising ISE for phosphorus detection was reported, but several literatures presented that the PVC-based membrane ISEs could be used to measure phosphate content in biological samples (Glazier, 1988; Carey, 1994; Liu, 1997, Fibbioli, 2000; Wroblewski, 2001). ISEs were used for soil nutrient detection in two directions: (1) Flow 
Injection Analysis (FIA) systems (Ruzicka, 1977; Hongbo, 1985; Ferreira, 1996), and (2) vehicle-based soil sensing systems (Adsett, 1991; V.I. Adamchuck, 2004). However, ISEs might not have been ready for real-time sensing applications because of their response delay (several minutes).

\subsection{Ion Selective Field Effect Transistor}

ISFET is the integration of an ISE and a field effect transistor (FET). The ion selective membrane is placed on the top of the insulator layer of the FET structure, so the threshold voltage of the ISFET can be chemically modulated and the measured voltage is related with the concentrations of a target ion. ISFETs have several advantages over ISEs, such as small dimensions, low output impedance, high signal-to-noise ratio, fast response and the ability to integrate mulit-ISFETs on one chip.

ISFETs were reported to detect soil ammonium (Oesch, 1981), nitrate (Van, 1994; J. Artigas, 2001) and potassium (Moss, 1975; 1978; Van, 1994; J. Artigas, 2001). Also, ISFETs were used in FIA systems and vehicle-based real-time soil sensing systems by researchers (A.U. Ramsing, 1980; Loreto, 1996). A successful automated system for soil $\mathrm{pH}$ mapping was reported to be tested under field conditions by Adamchuck et al. (2002). However, ISFET's high cost and inconsistent repeatability limited their wide extension use in practical systems.

\section{OPPORTUNITIES AND CHALLENGES}

Some emerging advanced techniques, such as MicroElectroMechanical System (MEMS), Microfluidics and Lab on valve (LOV), have been attempted for electrochemical detection in biological, chemical and medical fields and provide new ideas and good opportunities for electrochemical sensors from fabrication to measurement.

Multi-targets Simultaneous Detection. So far, most soil nutrient detection methods can only measure one target ion by using conventional electrochemical measurement due to an ion selective membrane, in theory, only selectively responding to one target ion. Electrochemical sensors can be integrated onto one chip as a sensor array to provide a feasible approach of multi-targets simultaneous detection. However, all the membranes developed for soil NPK detection did not respond to only one specific ion, but also to other interfering ions present in the analyte. One main challenge faced is the reliability of sensor array, that is, to avoid or diminish the interferences from other ions while using a sensor array for simultaneous detection.

Automation. Both ISEs and ISFETs have successfully been used in FIA systems. At present, micropumps and microvalves suitable for the 
development of accurate FIA systems are commercially available now. It has been possible to integrate one sensor or sensors with a FIA system to develop an automated ISE/ISFET-FIA system, even a microfluidic detection system on one chip. This kind of microsensors may be of automatic control and easily used even for non-trained users. However, the blocking of the microchannels by small particles should be considered.

Lower cost. Electrochemical sensors have the potential to be produced in batches to very small sizes by using MEMS-based microfabrication technology at a very low cost. The sensors can even be designed for one-off or multiple uses. Besides, small-sized sensors require small volume of reagents and samples, which can also reduce the cost in soil testing.

Rapidness. Time is a critical factor for soil nutrient detection since the variability of soil nutrient levels may be quite high over time (Sudduth et al., 1997). Due to complex soil pretreatment and chemical analysis, standard testing methods for soil NPK are time-consuming (in hours). Electrochemical sensors can rapidly respond to the target ions in minutes, suitable for in-field rapid detection.

\section{CONCLUSION}

The advantages of potentiometric electrochemical sensors are stimulating the interest of their applications in soil nutrient detection. They have potentials for automated multi-target rapid detection of soil nutrients. As such, they are also faced with the challenge from their reliability. Advanced engineering technologies have opened our mind and provided new approaches for soil testing to follow the KISS (Keep It Simple and Stupid) principle to treat the complex soil testing procedures with simpler methodology at a lower cost.

\section{ACKNOWLEDGEMENTS}

This research was supported by National 863 Project (2006AA10Z216).

\section{REFERENCES}

Ramsing A.U. et al. Anal. Chim. Acta, 1980, 118: 45

Adamchuk V.I. Feasibility of on-the-go mapping of soil nitrate and potassium using ionselective electrodes. Transaction of ASAE., 2002, 02, 1183

Adsett J.F., G.C. Zoerb. Automated field monitoring of soil nitrate levels. In: Automated agriculture for the 21st century, Mich.: ASAE Publ., 1991, 326-335 
Banwart W.J., et al. Commun. Soil Sci. Plant Anal., 1972, 3: 443-458

Carey C.M., W.B. Riggan. Cyclic polyamine ionophore for use in a dibasic-phosphate selective electrode. Anal. Chem., 1994, 66: 3587-3591

Dahnke W.C. Use of the nitrate specific ion electrode in soil testing. Soil Sci. and Plant Anal., 1971, 2(2): 73-84

Ferreira A.M.R., Lima J.L.C., Rangel A.O.S.S. Potentiometric determination of total nitrogen in soils by flow injection analysis with a gas-diffusion unit. Aust. J. Soil Res., 1996, 34: 503

Fibbioli M., M. Berger, F.P. Schmidtchen, et al. Polymeric membrane electrodes for monohydrogen phosphate and sulfate. Anal. Chem., 2000, 72(1): 156-160

Glazier S.A., M.A. Arnold. Phosphate-selective polymer membrane electrode. Anal. Chem., 1988, 60: 2540-2542

Hansen E.H., A.K. Ghose, J. Ruzicka. Flow injection analysis of environmental samples for nitrate using an ion-selective electrode. Analyst, 1977, 102: 705-713

Hongbo C., Hansen E.H., Ruzicha J., Evaluation of critical parameters for measurement of pH by flow injection analysis. Anal. Chim. Acta 169, 209 (1985) J. Artigas, A. Beltran, C. Jimenez, et al. Application of ion sensitive field effect transistor based sensors to soil analysis. Computers and Electronics in Agriculture, 2001, 31: 281-293

Liu D., W.C. Chen, R.H. Yang, et al. Polymeric membrane phosphate sensitive electrode based on binuclear organotin compound. Analytica Chimica Acta, 1997, 338: 209-214

Loreto A.B., Morgan M.T. Development of an Automated System for Field Measurement of Soil Nitrate. Transaction of ASAE, 1996

Mei Shourong. Soil Total Potassium Detection Using Potassium Electrode. Shanghai Agriculture Science and Technology, 1981, (01) (in Chinese)

Moss S.D., Janata J., Johnson C.C. Potassium, ion-selective field effect transistor. Anal. Chem., 1975, 47 (13): 2238

Moss S.D., Johnson C.C., Janata J. Hydrogen, calcium and potassium ion-sensitive FET transducers: a preliminary report. IEEE Trans. Biomed. Eng. 1978, BME-25, 49

National Bureau of Statistics of China. China Statistical Yearbook 2006. Beijing: China Statistics Press, 2006 (in Chinese)

Oesch U., Caras S. Field effect transistor sensitive to sodium and ammonium ions, Anal. Chem., 1981, 53: 1983

Ruzicka J., Hansen E.H., Zagatto E.A. Flow injection analysis. Part II: use of ion-selective electrodes for rapid analysis of soil extracts and blood serum. Determination of potassium sodium and nitrate. Anal. Chim. Acta, 1977, 88(1)

Simeonov V., et al. Anal. Letter, 1976, 9: 1025-1029

Sudduth K.A., J.W. Hummel, S.J. Birrell. 1997. Sensors for site-specific management, 183210, In: Pierce F.J. and E.J. Sadler (ed). The state of site-specific management for agriculture. Madison, Wisc. ASA-CSA-SSSA

Adamchuk V.I., J.W. Hummel, M.T. Morgan, et al. On-the-go soil sensors for precision agriculture. Computers and Electronics in Agriculture, 2004, 44:71-91

Van der Wal P.D., Van der Berg A., De Rooij N.F. Universal approach for the fabrication of $\mathrm{Ca}_{2-}, \mathrm{K}+$ and $\mathrm{NO}_{3}-$ sensitive membranes ISFETs. Sensors and Actuators, 1994, B18-19, 200

Wang Baoli, Qu Dong, Tian Hua, Simultaneous Determination of Sodium and Potassium in Soil Extract by Ion Selective Electrode, Journal of Northwest Sci-Tech University of Agriculture and Forestry (Natural Science Edition), 1992, 20(3): 130-134 (in Chinese)

Wroblewski W., K. Wojciechowski, A. Dybko, et al. Durable phophate-selective electrodes based on uranyl salophenes. Anal. Chim. Acta, 2001, 432: 79-88. 